Check for updates

Cite this: Phys. Chem. Chem. Phys., 2020, 22, 28191

Received 28th September 2020, Accepted 22nd November 2020

DOI: $10.1039 / \mathrm{d} 0 \mathrm{cp} 05110 \mathrm{a}$

rsc.li/pccp

\section{Effect of water on the electroresponsive structuring and friction in dilute and concentrated ionic liquid lubricant mixtures $\dagger$}

\author{
Georgia A. Pilkington, (D)*a Rebecca Welbourn, (D) ${ }^{\mathrm{b}}$ Anna Oleshkevych, ${ }^{\mathrm{a}}$ \\ Seiya Watanabe, (DD $\ddagger^{a}$ Patricia Pedraz, ${ }^{a}$ Milad Radiom, (D) a Sergei Glavatskih (D) ${ }^{\mathrm{cd}}$ \\ and Mark W. Rutland (ID *ae
}

\begin{abstract}
The effect of water on the electroactive structuring of a tribologically relevant ionic liquid (IL) when dispersed in a polar solvent has been investigated at a gold electrode interface using neutron reflectivity (NR). For all solutions studied, the addition of small amounts of water led to clear changes in electroactive structuring of the IL at the electrode interface, which was largely determined by the bulk IL concentration. At a dilute IL concentration, the presence of water gave rise to a swollen interfacial structuring, which exhibited a greater degree of electroresponsivity with applied potential compared to an equivalent dry solution. Conversely, for a concentrated IL solution, the presence of water led to an overall thinning of the interfacial region and a crowding-like structuring, within which the composition of the inner layer IL layers varied systematically with applied potential. Complementary nanotribotronic atomic force microscopy (AFM) measurements performed for the same IL concentration, in dry and ambient conditions, show that the presence of water reduces the lubricity of the IL boundary layers. However, consistent with the observed changes in the IL layers observed by NR, reversible and systematic control of the friction coefficient with applied potential was still achievable. Combined, these measurements provide valuable insight into the implications of water on the interfacial properties of ILs at electrified interfaces, which inevitably will determine their applicability in tribotronic and electrochemical contexts.
\end{abstract}

\section{Introduction}

Ionic liquids (ILs), due to their many unique properties, have received much interest across a wide ranging span of disciplines and application areas. In particular, owing to their inherent ionic nature, they are attractive candidates for many energy applications. For example, in fuel and solar cells, super-capacitors and electrochemical devices, as well as in lubrication, where they have the

\footnotetext{
${ }^{a}$ Division of Surface and Corrosion Science, School of Engineering Sciences in Chemistry, Biotechnology and Health, KTH Royal Institute of Technology, SE-100 44 Stockholm, Sweden. E-mail: georgiap@kth.se, mark@kth.se

${ }^{b}$ ISIS Facility, Rutherford Appleton Laboratory, STFC, Chilton, Didcot, OXON OX11 OQX, UK

${ }^{c}$ System and Component Design, Department of Machine Design, KTH Royal Institute of Technology, SE-100 44 Stockholm, Sweden

${ }^{d}$ Department of Electromechanical, Systems and Metal Engineering, Ghent University, B-9052 Ghent, Belgium

${ }^{e}$ Department of Material and Surface Design, RISE Research Institutes of Sweden, SE-100 44 Stockholm, Sweden

$\dagger$ Electronic supplementary information (ESI) available. See DOI: 10.1039/ d0cp05110a

\# Current address: Tokyo University of Science, Department of Mechanical Engineering, 6-3-1 Niijuku, Katsushika-ku, Tokyo 125-8585, Japan.
}

potential to enhance energy efficiency by facilitating active friction control by changing the surface charge to modify the boundary layer structure. ${ }^{1}$ However, one major challenge to the success of ILs in these applications is the omnipresence of water.

Many IL synthesis methods typically involve water, and even hydrophobic ionic liquids can absorb significant amounts from the atmosphere, making the presence of water, particularly for practical industrial purposes, almost inevitable. Previous studies on pure IL systems have shown that water can significantly influence the chemical and physical properties of ILs, including their electrochemical ${ }^{2}$ and rheological behaviours, ${ }^{3-5}$ as well as lubrication properties. ${ }^{6-9}$ Furthermore, in the case of many ILs explored for tribological purposes, water molecules have been shown to preferentially interact with the halogen-containing anions and, under high pressure and load conditions, produce toxic and corrosive halogen halides. ${ }^{10,11}$ Similar effects are also potentially detrimental for electrochemical applications, whereby the electrolysis of water can give rise to chemically reactive $\mathrm{H}^{+}$ions. ${ }^{12}$ For these reasons, in recent years there has been an increasing demand for halogen-free IL solutions.

A number of studies have investigated the effects of water on the friction and boundary lubrication structures of ILs. ${ }^{13}$ In the 
case of hygroscopic ILs the absorption of water has been shown to lead to a decrease in the IL viscosity, ${ }^{8}$ thus accelerating squeeze-out of the fluid film under applied load, and attributed to a weakening of the electrostatic interactions between the IL component ions. ${ }^{3}$ Conversely, water in hydrophobic ILs has been shown to increase adhesion and worsen lubrication performance. ${ }^{7}$ In a bilayer-forming IL system, the interpenetration of water molecules between the IL bilayers was also reported to result in an increased friction and ascribed to the water molecules inducing greater ordering of the ions resulting in a solidification effect. ${ }^{9}$ However, by and large, water predominantly has been shown to have a disrupting effect on the structuring of ILs at the solid-liquid interface. ${ }^{14-17}$

Control of the surface structuring and interfacial properties of ILs through electrical polarisation has already been demonstrated for a wide range of ILs and their solutions. For example, it has been shown that the frictional properties of ILs can be actively controlled by changing the surface charge to modify the boundary layer structure. ${ }^{18-21}$ Through choice of a polar solvent, it has also been established that the electro-responsivity of the IL boundary layers can be enhanced, whilst maintaining good lubrication properties. ${ }^{21,22}$

To the best of the authors' knowledge, previous studies that have investigated the effect of water on the interfacial structuring of ILs at electrified interfaces have been limited to the case of pure, halogenated ILs. Molecular dynamic (MD) simulations have shown that the water molecules in a humid halogenated IL are depleted from the electrode interface (compared to the bulk) at small charge densities, but enriched for larger ones. ${ }^{23}$ In the same study, the capacitance across the humid IL was predicated to be notably increased, particularly at positive potentials, which was attributed to the higher affinity of water towards the IL anions. This study thus predicts a strongly anisotropic water composition with electric potential in the near surface region.

Depending on the water content of the bulk, it has also been predicted that the presence of water can serve to either shrink or swell the interfacial ionic layer. ${ }^{24}$ At lower water fractions, the interfacial layers are found to become more compact, due to a local increase in the effective dielectric constant in the interfacial region, whilst at higher water fractions the layers swell due to the additional volume of the water molecules. In turn, such changes in the interfacial structuring of ILs have been predicted to lead to a reduction or increase in friction, respectively.

Such theoretical predictions have also been supported experimentally by differential capacitance, ${ }^{2}$ surface-enhanced infrared absorption spectroscopy (SEIRAS), ${ }^{25}$ surface force apparatus (SFA) ${ }^{7}$ and atomic force microscopy (AFM) measurements. ${ }^{17}$ However, in other cases, little effect on the ion distribution in the interfacial region near electrodes has been reported, but instead a notable acceleration of the potential-dependent cation/anion exchange observed and attributed to the reduction of the Coulombic interactions amongst the ions and between ions and the electrode surface. ${ }^{25}$ Such weakened interactions between the cation and anions are anticipated to also be the cause of the marked reductions in the stiffness of some IL layers in the presence of water, therefore lowering their load carrying capacity. ${ }^{7,26}$

Here, we have investigated the effect of water on the interfacial structuring of a tribologically relevant, non-halogenated IL, which has previously been reported to provide excellent lubrication. ${ }^{27}$ Furthermore, when dispersed in a base fluid, this IL has been shown to undergo distinct restructuring under the influence of an electric field, resulting in prominent tribotronic properties. $^{21,22,28,29}$ Prior to this study, the same IL-propylene carbonate (PC) solutions, without the addition of water, showed clear electro-responsive structuring for different applied potentials, which was influenced also by the bulk IL concentration. ${ }^{21}$ In particular, the results suggested that, below a critical concentration, the IL cations exhibit a specific interaction with the gold surface, hindering the responsivity at negative biases.

As demonstrated in previous neutron reflectivity (NR) studies, ${ }^{21,22}$ to improve sensitivity to changes in the interfacial region, we choose to contrast match the scattering length density (SLD) of the bulk solution to that of the gold electrode $\left(\mathrm{SLD} \approx 4.65 \times 10^{-6} \AA^{-2}\right)$. To create contrast with the interfacial region, which is anticipated to be predominantly populated with the IL ions, ${ }^{21}$ perdeuterated water $\left(\mathrm{D}_{2} \mathrm{O}\right)$, which has a large $\operatorname{SLD}\left(6.34 \times 10^{-6} \AA^{-2}\right)$, was added to the IL solutions, rather than hydrogenated water. SLD profiles, obtained from model fits to the reflectivity data, are compared with those previously reported for dry solutions, without the addition of water, for the same bulk IL concentrations and solvent. ${ }^{21}$ To determine the influence of water on the interfacial friction of such IL layers, complementary nanotribotronic AFM measurements were also performed in dry and ambient conditions at one concentration of the IL in PC.

Together the results reveal the presence of water strongly influences the electroactive structuring and lubrication properties of the IL layers at the gold electrode interface. However, the distinct ability to systematically tune the interfacial frictional properties with an applied potential in dry conditions is demonstrated to remain feasible even under ambient conditions, where significant water levels are present.

\section{Materials and Methods}

\subsection{Materials and solution preparation}

The IL trihexyl(tetradecyl)phosphoniumbis(mandelato)-borate, $\left[\mathrm{P}_{6,6,6,14}\right][\mathrm{BMB}]$ ( $c f$. Fig. 1) was synthesised at Luleå University of Technology. ${ }^{30}$ This IL was chosen due to its excellent lubrication and electro-responsive properties..$^{11,28,30,31}$ Prior to all measurements, the pure $\left[\mathrm{P}_{6,6,6,14}\right][\mathrm{BMB}]$ was baked under vacuum at a pressure of 12 mbar and a maximum temperature of $60{ }^{\circ} \mathrm{C}$ for approximately $72 \mathrm{~h}$ to remove traces of solvents and water. The solvents propylene carbonate (anhydrous, 99.7\%; Sigma Aldrich), 1,2-propylene- $\mathrm{d}_{6}$ carbonate (99.5 atom\% D; Qmx Laboratories Ltd) and $\mathrm{D}_{2} \mathrm{O}$ (99.9\% atom D; Sigma Aldrich) were used as received.

All IL solutions were prepared by weighing the IL and adding the appropriate volume of the solvents and $\mathrm{D}_{2} \mathrm{O}$. In order to minimise ambient water contamination, the solutions were 


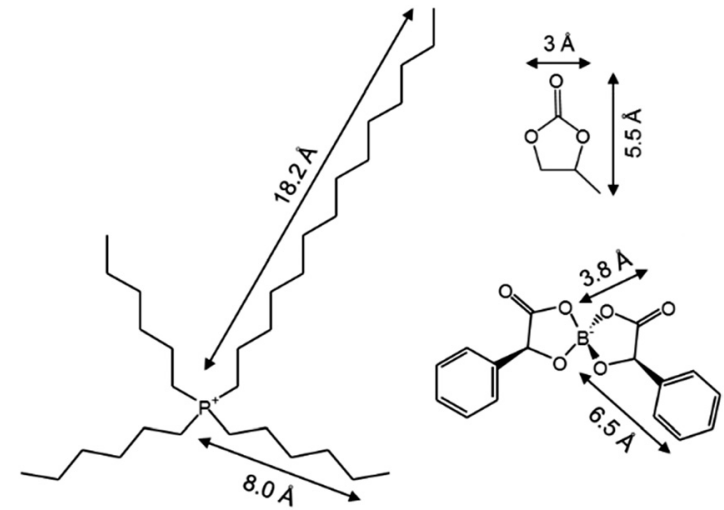

Fig. 1 Molecular structure and dimensions of the ionic liquid trihexyltetradecylphosphoniumbis(mandelato)borate, $\left[\mathrm{P}_{6,6,6,14}\right][\mathrm{BMB}]$ and the solvent propylene carbonate $(\mathrm{PC})$

prepared under a dry, nitrogen atmosphere (R.H. $<10 \%)$. To ensure mixing, each solution was sonicated for at least 10 minutes until homogeneous (by eye) and used immediately, or stored under nitrogen. The concentrations of the solutions are presented in terms of IL weight percentage (\% w/w) in PC. 5 and $20 \% \mathrm{w} / \mathrm{w}\left[\mathrm{P}_{6,6,6,14}\right][\mathrm{BMB}]$ in $\mathrm{PC}$ correspond to 0.7 and $3 \mathrm{~mol} \%$, or 0.08 and $0.3 \mathrm{M}$, respectively. These two concentrations are expected to represent the dilute (Debye-Hückel) and concentrated regimes. ${ }^{32}$

For NR measurements, the bulk SLD of all solutions was contrast matched to that of gold by using appropriate amounts of hydrogenated and deuterated PC ( $c f$. Table 1). ATR-IR measurements (Nicolet 8700 FT IR Spectrometer, Thermo Scientific), were performed on the IL solutions before and after the NR measurements to obtain molecular vibration information. A diamond crystal was used for the ATR prism. A droplet of IL solution was placed on the top of the ATR prism. The spectral resolution was fixed to $4 \mathrm{~cm}^{-1}$ and number of accumulations was 64 , corresponding to a sampling time of $1 \mathrm{~min}$. The IR spectra for the solutions used for the NR measurements are provided in the ESI $\dagger$ and did not show any evidence of electro-induced breakdown of the IL ( $c f$. ESI, $\dagger$ Fig. S1).

In order to establish the amount of water in the dry solvent, PC, Karl Fischer measurements were performed. The amount of water was determined to be $45.8 \mathrm{ppm}$. Equivalent, direct measurements of the water content of the orthoborate ILs or their solutions by Karl Fischer is not possible, as they react with the Karl Fischer reagents in a side reaction which leads to an incorrect water content. For this reason, the amount of water in the dry IL solutions was estimated from FT-IR spectra by

Table 1 Scattering length densities of different molecular species

\begin{tabular}{lc}
\hline Species & SLD $\left(\times 10^{-6} \AA^{-2}\right)$ \\
\hline$\left[\mathrm{P}_{6,6,6,14}\right][\mathrm{BMB}]$ & 0.5 \\
{$\left[\mathrm{P}_{6,6,6,14}\right]^{+}$} & -0.4 \\
{$[\mathrm{BMB}]^{-}$} & 2.68 \\
$\mathrm{PC}$ & 1.53 \\
$\mathrm{~d}_{6}-\mathrm{PC}$ & 5.89 \\
$\mathrm{D}_{2} \mathrm{O}$ & 6.34
\end{tabular}

correlating the peak intensity of $\mathrm{O}-\mathrm{H}$ stretching mode with actual water amount measured by Karl Fischer method. Based on the spectra presented in Fig. S5 ( $c f$. ESI $\dagger$ ), the amount of water in the 5 and $20 \%$ w/w dry IL solutions is estimated to be 71 and $31 \mathrm{ppm}$, respectively.

\subsection{Preparation of gold surfaces}

Gold thin $(\sim 170 \AA)$ films were prepared by electron beam evaporation (Auto 306, Edwards High Vacuum International, Wilmington, MA) atop silicon substrates and used as working electrodes. For the NR measurements, the films were deposited on $10 \mathrm{~mm}$ thick $(50 \times 50 \mathrm{~mm})$ polished silicon (100) blocks (Sil'tronix Silicon Technologies, France). For the AFM measurements, the films were coated on top of thin $(0.5 \mathrm{~mm})$ polished silicon (100) wafer chips (University Wafer, Boston, MA, US). To ensure good adhesion between the $\mathrm{Si}$ and $\mathrm{Au}$, the $\mathrm{Si}$ surfaces were pre-coated with a titanium $(\sim 30 \AA)$ adhesion layer. Prior to deposition, the Si surfaces were cleaned in Piranha solution (prepared as a 3:1 mixture of concentrated sulphuric acid and hydrogen peroxide), rinsed with Milli-Q water and dried with filtered $\mathrm{N}_{2}$. Before all measurements, the coated surfaces were rinsed with filtered ethanol, blow dried with nitrogen and exposed to UV/ozone for 10 minutes. Prior to all solution NR measurements, a short length of insulated copper wire was adhered to one corner of each gold surface using a conductive epoxy (CW400, Chemtronics), which was cured in an oven at $120{ }^{\circ} \mathrm{C}$ for 30 minutes before repeating the same cleaning procedure.

\subsection{Neutron reflectivity measurements and analysis}

The NR measurements were performed on the INTER reflectometer at the ISIS Facility, Rutherford Appleton Laboratory, UK. All reflectivity measurements were performed using time-offlight mode, in a horizontal geometry. The neutron specular reflectivity $R$ (the ratio of the reflected beam to the incident beam intensity) was measured as a function of the momentum transfer normal to the interface $Q_{z}=4 \pi \sin \theta / \lambda$, where $\theta$ is the angle of incidence and $\lambda$ is the wavelength of the neutron beam.

Reflectivity measurements were performed on the IL solutions using a custom-made electrochemical NR cell. ${ }^{22}$ The neutron beam was directed through the Si block and reflected from the gold interface, with the reflected beam traversing through the other side of the block, and exiting to the detector. slits were used to define the beam size and ensure the beam footprint was within the area of the gold surface that was covered by IL. For each potential studied, the cell was first allowed to stabilise for 30 minutes. The reflectivity measurements on the IL solutions were measured at two incident angles $\left(0.5^{\circ}\right.$ and $\left.1.8^{\circ}\right)$, providing a $Q$-range of $0.0065-0.22 \AA^{-1}$. The resolution $(\mathrm{d} Q / Q)$ for the measurements was selected to be around $4 \%$. The scattering data were reduced in Mantid $^{33}$ using standard procedures. The data were normalised to a transmission measurement through the silicon block and the measurements from the two angles were stitched together to form one profile. 
The data were fitted using a slab model based on the reflections from a stratified medium; i.e. to a model consisting of a series of layers, each with an associated thickness, roughness and scattering length density (SLD - composition). This was performed using the software package GenX. ${ }^{34}$ The thicknesses, SLDs and roughnesses of the substrates native $\mathrm{SiO}_{2}$ layer, $\mathrm{Ti}$ adhesion layer and $\mathrm{Au}$ layer atop a $\mathrm{Si}$ block were characterised beforehand by performing reflectivity measurements on the blocks in air ( $c f$. ESI, $\dagger$ Fig. S2 and Table S1).

The gold interfacial roughness $(\sim 10 \AA)$ was found to be comparable to the molecular dimensions of the IL ( $c f$. Fig. 1), so the usual Nevot-Croce approach ${ }^{35}$ to the roughness is not suitable at this interface. Instead, this interface has been modelled using a micro-slabbing approach: the interfacial region is split into a series of $1 \AA$ thick slabs of $0 \AA$ roughness. The SLD of each microslab is determined using a normal distribution of the volume fraction of $\mathrm{Au}$ and the first IL layer to describe a smooth transition between these regions. The only fittable parameter was the thickness of this interfacial region $\left(t_{\mathrm{i}}\right)$, which replaces the gold interfacial roughness parameter. The cumulative distribution function in SciPy was used to calculate the normal distribution, with the scale set to $3 t_{\mathrm{i}} / 20$ to ensure the volume fraction approached 0 and 1 at the edges of $t_{\mathrm{i}}$. This parameter along with the coupled parameter of the Au thickness and the bulk IL SLD were fitted using the $0 \mathrm{~V}$ condition and fixed for all subsequent conditions. All other parameters related to the substrate (i.e. the Ti and $\mathrm{SiO}_{2}$ thickness, roughness and SLD), were fixed to those obtained from the substrate reflectivity measurements in air. The thickness, roughness and SLD values of the IL layers were allowed to vary between potentials. Best fits to the reflectivity data were determined by the lowest logarithm of the figure of merit (FOM) for a minimum number of layers.

Details regarding the cell design have been reported previously in a related study of IL-solvent mixtures. ${ }^{22}$ However, briefly, before assembling the cell, the conductive glass (coated with a fluorine-doped tin oxide, FTO; NSG TEC A7, Pilkington, UK) used as counter electrode, as well as all the other cell components, were sonicated in $2 \%$ Hellmanex for 30 minutes, before being rinsed thoroughly with Milli-Q water and absolute ethanol, and dried with filtered $\mathrm{N}_{2}$. For the measurements presented here, a PTFE gasket of $0.5 \mathrm{~mm}$ thickness was used to separate the working (gold films) and counter electrodes. In order to minimise ambient water contamination, the cell was assembled inside a polyethylene glove bag under a dry, nitrogen atmosphere (R.H. $<10 \%$ ). The IL solutions were injected into the bottom port of the cell through PTFE tubing using a glass Luer syringe. Once mounted on the beamline, a Metrohm Autolab (PGSTAT204) potentiostat was used to apply potentials across the cell. During all NR measurements, the potential and current across the cell were continually recorded and monitored. By integration of the current during the stabilisation time, and dividing it by the working electrode surface area, estimates of the surface charge density for each condition were made ( $c f$. ESI, $\dagger$ Tables S2-S4). The surface area of the gold (working electrode) was defined by the PTFE gasket used to seal the solution and is estimated to be $17.6 \mathrm{~cm}^{2}$.

\subsection{Cyclic voltammetry and charge density measurements}

Cyclic voltammetry (CV) measurements were performed across each IL solution in the electrochemical NR cell after the reflectivity measurements ( $c f$. Section 2.3.2.) to establish that no faradaic events occurred within the experimental potential window studied, as well as determine the reversibility of the charge transfer. The potential was cycled between -1.5 and $+0.25 \mathrm{~V}$ several times at different rates (6 and $10 \mathrm{mV} \mathrm{s}^{-1}$ ) and the current response monitored. Representative CV plots at $10 \mathrm{mV} \mathrm{s}^{-1}$ are shown for each solution in the ESI, $\dagger$ ( $c f$. Fig. S3, ESI $\dagger)$. No changes were observed over multiple sequential cycles, indicating the absence of any electrochemical reactions within the electrochemical window of study. In addition, cyclic voltammetry measurements were performed across freshly prepared IL solutions with $100 \mathrm{mM}$ ferrocene to obtain the half wave potential $\left(E_{1 / 2}\right)$ of the ferrocene/ ferrocenium $\left(\mathrm{Fc}^{+} / \mathrm{Fc}\right)$ redox pair. This internal standard allows for comparison between different electrochemical cells or setups.

\subsection{Friction force measurements}

Electrochemical friction force measurements were performed using a JPK Nanowizard 3 Ultraspeed atomic force microscope (AFM) equipped with a JPK ECCellTM electrochemical cell with a Kalrez gasket and o-ring. Friction was measured between a sharp Si AFM tip (radius $R=7 \mathrm{~nm}$; AC204TS, Olympus) and a gold coated silicon wafer which acted as a working electrode. The potential was applied using a PGU BI-1000 potentiostat (Ingenieurbüro Peter Schrems (IPS), Germany). Pt wires were used as counter (CE) and reference (RE) electrodes. Ferrocene was used as an internal standard to determine the potential of the Pt pseudo-reference electrode $v s . E_{1 / 2}\left(\mathrm{Fc}^{+} / \mathrm{Fc}\right)$, as described in Section 2.4.

Prior to the measurements, the electrochemical cell was disassembled and cell parts thoroughly cleaned using acetone, isopropanol (IPA) and absolute ethanol, before being dried with filtered nitrogen. The AFM tip and gold surface were also rinsed with absolute ethanol and irradiated in an ultraviolet (UV) ozone cleaner (PCE-44-LD; MTI Corporation, US) for $10 \mathrm{~min}$ utes. Friction measurements were performed over a scan size of $500 \mathrm{~nm}$ and at a scan rate of 1,6 and $12 \mu \mathrm{m} \mathrm{s}^{-1}$. The lateral deflection, measured as changes in the lateral voltage signal $\Delta V$, were averaged over 10 trace and retrace cycles at each normal load. The averaged lateral signals were converted to friction force using the following equation:

$$
F_{\mathrm{f}}=\frac{\Delta V k_{\phi}}{2 h_{\mathrm{eff}} \delta},
$$

where $k_{\phi}$ is the torsional spring constant, $h_{\text {eff }}$ is the effective height of the AFM tip (length plus half the thickness of the cantilever) and $\delta$ is the lateral reflection sensitivity. ${ }^{36,37} k_{\phi}$ was calculated using the Hybrid method developed by ÁlvarezAsencio et $a .^{38}$ The lateral sensitivity of the AFM photodiode was corrected for the refractive index $(\mathrm{RI}=1.424)$ of the solutions, ${ }^{39}$ which was determined using an Abbe refractometer (RMT, Optech). The friction coefficient $\mu$ was obtained for each data set using modified Amontons' Law, where $\mu$ is 
extracted from the gradient of the friction force versus the normal load $F_{\mathrm{N}}$ in the linear region.

The measurements for the dry solution have been reported recently. ${ }^{21}$ To minimise water absorption during the measurements in "dry" conditions, the AFM measurements were performed under an Argon atmosphere. For the AFM measurements in ambient conditions (R.H. 22\%), the solution was allowed to equilibrate with the atmosphere for 30 minutes. IR measurements performed show that a $20 \% \mathrm{w} / \mathrm{w}$ solution of $\left[\mathrm{P}_{6,6,6,14}\right][\mathrm{BMB}]$ in PC when exposed to similar ambient conditions becomes saturated with water after 10 minutes ( $c f$. ESI, $\dagger$ Fig. S5). The water uptake was estimated to be $0.08 \% \mathrm{w} / \mathrm{w}$ from the water peak in the 3500-3700 $\mathrm{cm}^{-1}$ wavelength range using the same method described for the dry IL solutions ( $c f$. Section 2.1). The open circuit potential (OCP) of the dry and ambient solutions was $-160 \mathrm{mV}$. All potentials were applied for 30 minutes prior to each set of friction measurements. The average friction coefficient for PC (without potential) was $\sim 0.14$ at $6 \mu \mathrm{m} \mathrm{s}^{-1}$ (cf. Fig. 6b).

\section{Results and discussion}

\subsection{Neutron reflectivity}

Neutron reflectivity measurements were conducted for three IL- $\mathrm{D}_{2} \mathrm{O}$ solutions in $\mathrm{PC}$ at four applied potentials $(0,-1,+0.25$, $-1.5 \mathrm{~V})$ using a home-built electrochemical liquid cell. ${ }^{22} \mathrm{D}_{2} \mathrm{O}$, which has a high SLD, was used instead of $\mathrm{H}_{2} \mathrm{O}$ to provide contrast with the IL layers which are expected to be of lower SLD ( $c f$. Table 1). For all solutions and potential conditions studied, pronounced Kiessig fringes were observed in the reflectivity curves, signifying a region of lower SLD than that of the bulk IL solution (which was contrast matched to that of the gold). Thus, in all cases a distinct IL boundary layer was formed at the gold surface.

Fig. 2 shows the Fresnel normalised reflectivity curves for the 5 and $20 \%\left[\mathrm{P}_{6,6,6,14}\right][\mathrm{BMB}]$ in $\mathrm{PC}$ with $0.5 \% \mathrm{D}_{2} \mathrm{O}$ (a corresponding plot for $20 \%\left[\mathrm{P}_{6,6,6,14}\right][\mathrm{BMB}]$ in $\mathrm{PC}$ with $1 \% \mathrm{D}_{2} \mathrm{O}$ is provided in the $\mathrm{ESI}, \dagger c f$. Fig. S4). As the potential was varied, the amplitude of the fringe maxima and minima increased and decreased, depending on the applied potential bias. The relative changes in the reflectivities with respect to $0 \mathrm{~V}$ can be seen in the asymmetry plots shown in the Fig. 2 insets. In general, negative biases (blue and green markers) led to an increased fringe amplitude, revealing that the contrast with gold was more pronounced and thus the average SLD of the interfacial region became lower. This effect was then reversed when a positive potential was applied (red markers).

To obtain a more detailed understanding of the origins of these changes in the interfacial region (i.e. in terms of relative changes in the populations of cations, anions and $\mathrm{D}_{2} \mathrm{O}$ molecules), it is necessary to fit the reflectivity curves. Here we have employed a slab model, whereby the interfacial region is separated into a minimum number of layers in order to provide a best fit to the experimental reflectivity data ( $c f$. Section 2.3). The variation in the SLD obtained from these best fit models can then be compared as a function of the distance $(z)$ from the gold electrode interface. The parameters fitted for each layer
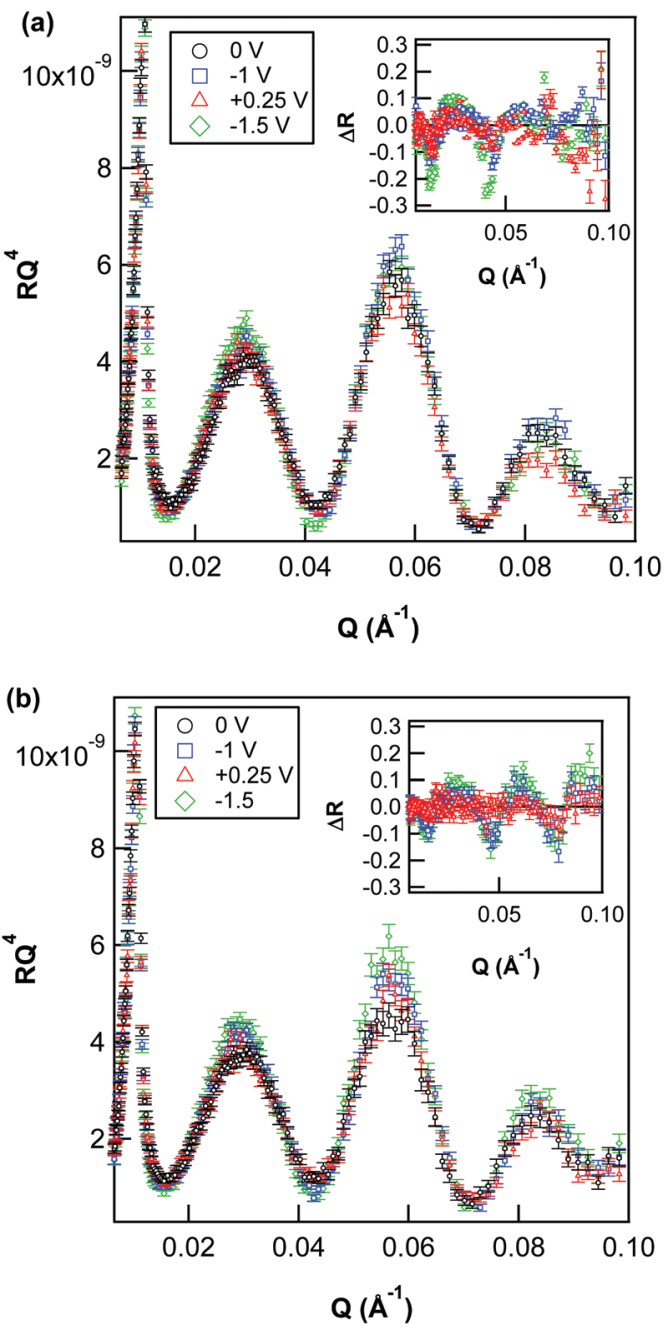

Fig. 2 Fresnel normalised reflectivity for (a) 5 and (b) $20 \% \mathrm{~W} / \mathrm{W}$ $\left[\mathrm{P}_{6,6,6,14}\right][\mathrm{BMB}]$ in $\mathrm{PC}$ with $0.5 \% \mathrm{w} / \mathrm{w} \mathrm{D}_{2} \mathrm{O}$ at gold electrode surface for different applied potentials (indicated in the legend). The insets show asymmetry plots $\Delta R=\left[R^{\vee}(Q)-R^{0}(Q)\right] /\left[R^{\vee}(Q)+R^{0}(Q)\right]$ highlighting the changes in the non-zero potential reflectivities $\left(R^{\vee}(Q)\right)$ with respect to those at $0 \vee\left(R^{0}(Q)\right)$.

are presented in the ESI $\dagger$ ( $c f$. Tables S5-S7). The far left of the SLD profiles corresponds to the bulk IL solution, whilst the far right handside corresponds to the gold interface. Further details of these models are discussed in the following sections.

3.1.1 $5 \%\left[\mathbf{P}_{6,6,6,14}\right][\mathrm{BMB}]+\mathbf{0 . 5 \%} \mathrm{w} / \mathbf{w} \mathrm{D}_{2} \mathbf{O}$. Reflectivity curves for $5 \%\left[\mathrm{P}_{6,6,6,14}\right][\mathrm{BMB}]+0.5 \% \mathrm{w} / \mathrm{w} \quad \mathrm{D}_{2} \mathrm{O}$ at different applied potentials at a gold electrode interface are shown in Fig. 3a, along with corresponding theoretical fits. SLD profiles corresponding to these best fit reflectivity profiles are shown in Fig. 3b. For comparison, the previously reported best fit SLD profiles for a dry IL-PC solution (without added water) at the same bulk IL concentration are shown in the Fig. 3b inset. Notably the SLD profiles from the dry solution revealed a single layer that was broadly similar for 0 and $-1.5 \mathrm{~V}(-1 \mathrm{~V}$ was not studied), but became thicker at the positive potential, $0.25 \mathrm{~V}$. In contrast, the SLD models for the $5 \% \mathrm{w} / \mathrm{w}$ solution with $0.5 \% \mathrm{w} / \mathrm{w} \mathrm{D}_{2} \mathrm{O}$, reveal a thicker interfacial region, of overall higher SLD, and thus suggest 

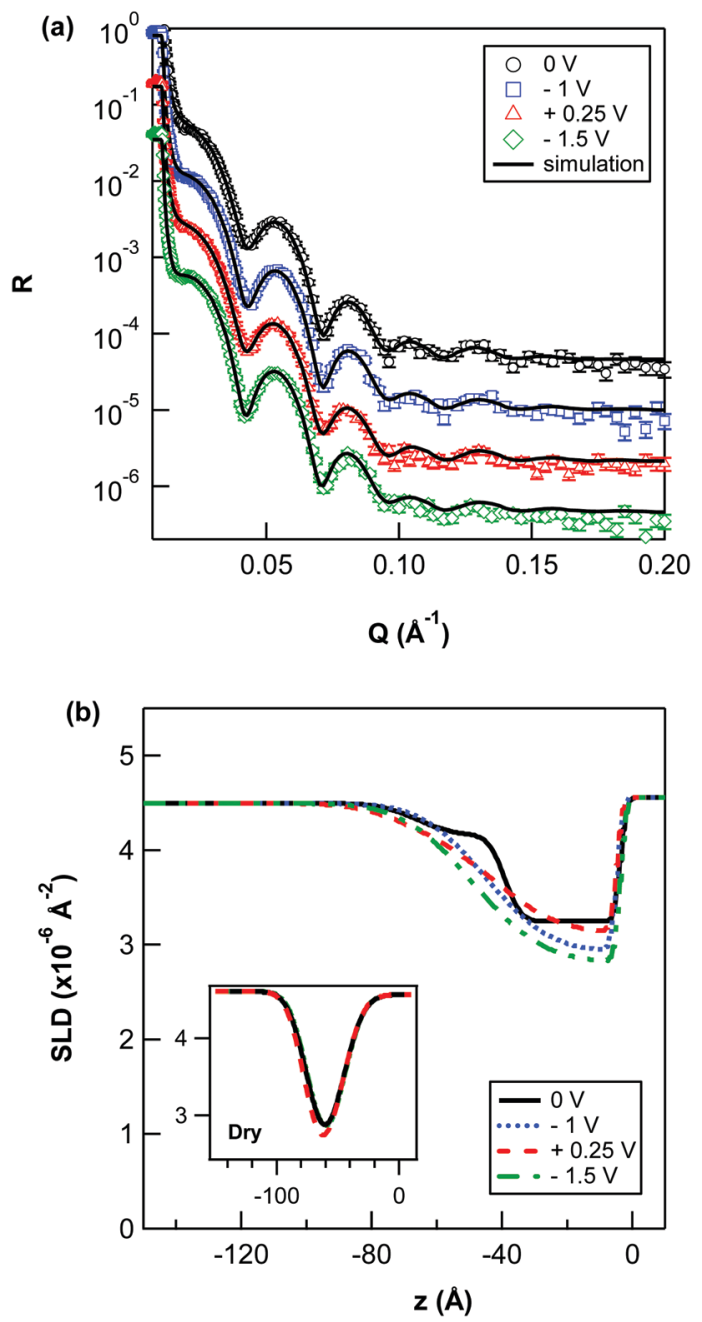

Fig. 3 (a) Reflectivity of $5 \% \mathrm{w} / \mathrm{w}\left[\mathrm{P}_{6,6,6,14}\right][\mathrm{BMB}]$ in $\mathrm{PC}$ with $0.5 \% \mathrm{D}_{2} \mathrm{O}$ at gold electrode surface for different applied potentials (indicated in the legend). The symbols show experimental data. The solid lines represent simulated reflectivity obtained from SLD model fits. The reflectivity datasets and simulated fits have been offset in the $y$-axis for clarity. (b) Corresponding model SLD profiles to the fits to the reflectivity curves. The potentials were applied as listed in the legend. The inset shows previously reported SLD profiles for "dry" solution of $5 \% \mathrm{w} / \mathrm{w}$ $\left[P_{6,6,6,14}\right][B M B]$ in $P C$ on gold from ref. 21 at $0,-1.5$ and $0.25 \mathrm{~V}$. The curve for $-1.5 \mathrm{~V}$ is largely similar that as $0 \mathrm{~V}$ at the scale used so is not visible.

that the presence of water leads to a swelling of the interfacial region. It is also notable from the SLD profiles, that the interfacial region undergoes more significant changes with applied potential.

Direct comparison with simulations in pure ILs is complicated as the SLD values here suggest that the interfacial region is likely to contain a significant fraction of the solvent, PC, which has a large dielectric constant, comparable to that of water. Thus arguments of a local increase in dielectric constant $^{24}$ in the interfacial region with water need to be tempered, since the relevant increase in dielectric constant will be commensurately less. Nonetheless, the former observation is consistent with an overall higher interfacial water content.

With respect to the second observation, it has previously been predicted that the different packing abilities of IL ions and charge distributions can lead to anisotropy with respect to the water distribution. ${ }^{23}$ Similarly, in the case of the IL studied here, the anions are expected to pack more poorly, due to their bulky and rigid structure, thus leaving more interstices to be filled by water. Moreover, atomistic MD simulations of the solvation structures of the same (pure) and related ILs with water ${ }^{40}$ have previously revealed water molecules preferentially coordinate to the central polar segments in the orthoborate $[\mathrm{BMB}]^{-}$anions. Here, the SLD profiles similarly point to a coordination between the anion and water.

For $0 \mathrm{~V}$ and $+0.25 \mathrm{~V}$, a 2-layer model was found to provide the best fit to the reflectivity data, suggesting the formation of distinct inner and outer layers ( $c f$. Table S5, ESI $\dagger$ ). Conversely, for the negative potentials, a single layer provided the best fit. With applied potential, the SLD of the minimum next to the gold interface clearly decreased for the negative potentials and increased for the positive potential, whilst the outer layer somewhat followed an opposite trend ( $c f$. Table S5, ESI $\dagger$ ). Upon application of $-1.5 \mathrm{~V}$, the thickness of the low SLD region next to the interface also notably increased, pointing towards a possible tendency of the cation to adopt a more perpendicular orientation with respect to the electrode interface.

Similar ionic re-orientation behaviours have been previously predicted by Monte Carlo simulations to take place via translations and rotations in pure IL systems at high potential electrode interfaces to increase the fraction of charged groups at the surface. $^{41}$ Meanwhile, recent sum frequency generation (SFG) measurements have shown the orientation of the aromatic ring of imidazolium cations to be surface charge dependent. ${ }^{42,43}$

3.1.2 20\% $\left[\mathbf{P}_{6,6,6,14}\right][\mathrm{BMB}]+\mathbf{0 . 5 \%} \mathbf{w} / \mathbf{w} \mathbf{D}_{2} \mathbf{O}$. Reflectivity curves for $20 \%\left[\mathrm{P}_{6,6,6,14}\right][\mathrm{BMB}]+0.5 \% \mathrm{w} / \mathrm{w} \mathrm{D}_{2} \mathrm{O}$ at different applied potentials at a gold electrode interface are shown in Fig. 4a, along with corresponding theoretical fits. SLD profiles corresponding to these best fit reflectivity profiles are shown in Fig. 4b. For comparison, previously reported SLD profiles for an equivalent dry solution are shown in the inset of Fig. $4 \mathrm{~b}$. In contrast to the $5 \% \mathrm{w} / \mathrm{w}$ dry solution ( $c f$. Fig. $2 \mathrm{~b}$ inset), the dry $20 \% \mathrm{w} / \mathrm{w}$ solution was revealed to form a thick, IL-rich interfacial layer, which varied significantly in thickness and overall SLD, depending on the potential bias.

Here, the SLD profiles presented in Fig. $4 \mathrm{~b}$ reveal that the addition of $0.5 \% \mathrm{w} / \mathrm{w} \mathrm{D}_{2} \mathrm{O}$ causes a stratification or partitioning of the IL boundary region at the gold interface, not previously observed for the dry solution. This is most pronounced at $0 \mathrm{~V}$, where the SLD profile resembles a decaying oscillatory function extending into the bulk from the electrode interface, fitted as 3 layers. More specifically, this leads to the detection of a interstitial layer, not present for other potentials, of high SLD, and thus indicative of a high water content. Interestingly, a water rich layer has been observed previously for simulations of another pure, IL at a neutral electrode interface under humid conditions. ${ }^{23}$ While the reasons for this layer were not clear, and that the system is rather different, one can speculate as to whether this is a more general phenomenon.

At non-zero applied potentials the oscillatory-like function observed at $0 \mathrm{~V}$ is significantly dampened. Instead the interfacial 

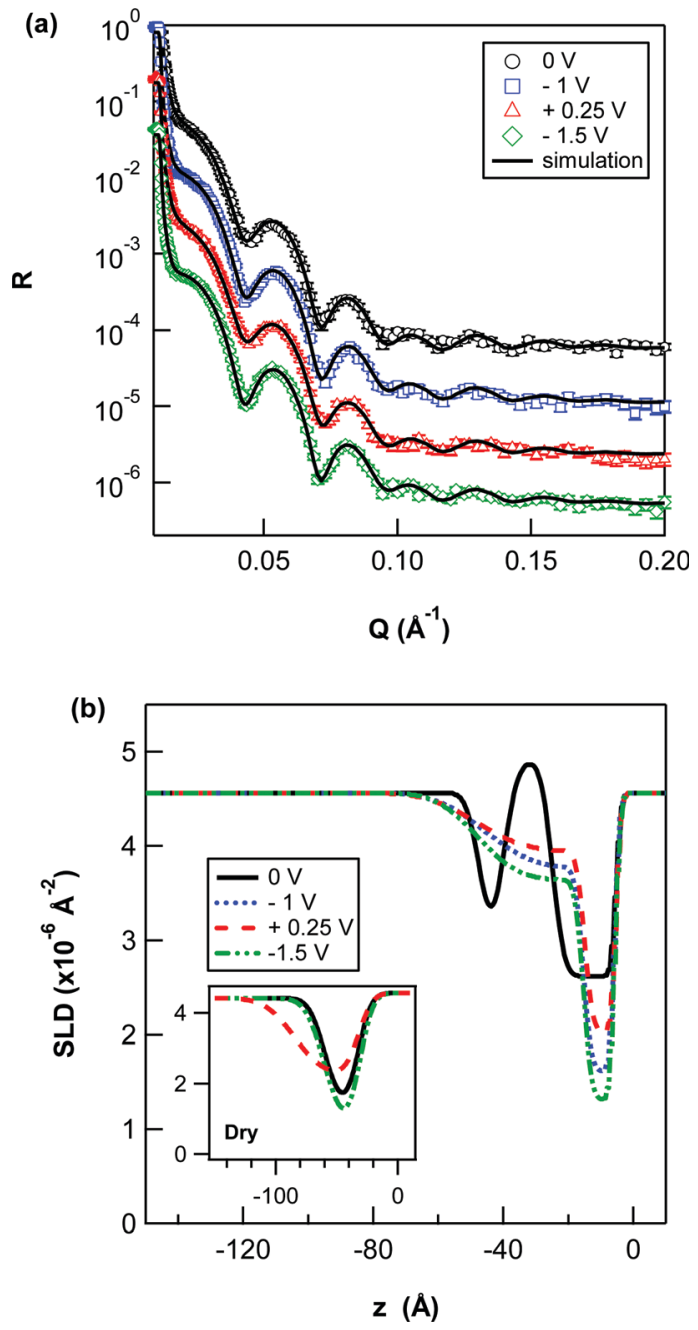

Fig. 4 (a) Reflectivity of $20 \% \mathrm{w} / \mathrm{w}\left[\mathrm{P}_{6,6,6,14}\right][\mathrm{BMB}]$ in $\mathrm{PC}$ with $0.5 \% \mathrm{D}_{2} \mathrm{O}$ at gold electrode surface for different applied potentials (indicated in the legend). The symbols show experimental data. The solid lines represent simulated reflectivity obtained from SLD model fits. The reflectivity datasets and simulated fits have been offset in the $y$-axis for clarity. (b) Corresponding model SLD profiles to the fits to the reflectivity curves. The potentials were applied as listed in the legend. The inset shows previously reported SLD profiles for "dry" solution of $20 \% \mathrm{w} / \mathrm{w}$ $\left[P_{6,6,6,14}\right][B M B]$ in $P C$ on gold from ref. 21

region is best fit with a 2 layer model, consisting of a deep SLD minimum next to the electrode interface, followed by a weaker, second minimum of higher SLD. Contrary to that observed for the dry solution, the total thickness of the interfacial region did not vary significantly with applied potential.

For all non-zero potentials, the SLD of the IL inner layer systematically varies depending on the applied potential bias and magnitude. Consistent with a higher anion fraction ( $c f$. Table 1), the SLD of the inner layer increases at the positive potential, whilst the SLD of the minimum decreases with increasingly negative potential, revealing a clear increase of the cation at the interface. The SLD of the second, outer layer also follows the same trend and thus suggests a "crowding structure".

Previously, theoretical ${ }^{44}$ and experimental ${ }^{45}$ studies have similarly demonstrated that, when the electrode polarisation increases, the interfacial structure of ILs undergoes a transition from overscreening the surface charge (by a single monolayer of counterions) to crowding of counterions (more than a single monolayer). However, here, there is also clearly a dramatic change in the interfacial ordering in the presence of water.

In contrast to the dilute $5 \% \mathrm{w} / \mathrm{w}$ solution, the SLD profiles imply that there is a lower solvent concentration at the interface and thus it can be argued that the presence of water leads to a local increase in the dielectric properties of this region. ${ }^{24}$ This modification to the Coloumbic interactions in turn appears to favour a more discrete layering of the ions, leading to the observed stratification of the IL boundary region.

3.1.3 $20 \%\left[\mathbf{P}_{\mathbf{6}, \mathbf{6}, \mathbf{6}, \mathbf{1 4}}\right][\mathrm{BMB}]+\mathbf{1} \% \mathbf{w} / \mathbf{w} \mathbf{D}_{2} \mathbf{O}$. Reflectivity curves for $20 \%\left[\mathrm{P}_{6,6,6,14}\right][\mathrm{BMB}]+1 \% \mathrm{w} / \mathrm{w} \mathrm{D}_{2} \mathrm{O}$ at different applied potentials at a gold electrode interface are shown in Fig. 5a, along with corresponding theoretical fits. SLD profiles corresponding to these best fit reflectivity profiles are shown in Fig. 5b. Overall, the SLD changes between different applied potentials were clearly more remarkable than for $0.5 \% \mathrm{D}_{2} \mathrm{O}$.

From the SLD profiles in Fig. $5 b$, it can be seen that similarly to $20 \% \mathrm{w} / \mathrm{w}+0.5 \% \mathrm{D}_{2} \mathrm{O}$, the IL forms a distinct minimum next to the gold interface, followed by a broader, more diffuse second layer. At the negative potentials, the SLD of the inner layer is notably lower for the negative potentials, implying a higher cation content. Although direct comparison with other, pure IL systems is challenging, an enhanced depletion of anions near the gold interface with increased water concentration is consistent with previous theoretical predictions for a negative surface charges. ${ }^{23}$

Upon reversing the potential bias to $+0.25 \mathrm{~V}$, the SLD profile changes significantly to a dampened oscillatory-like function, comparable to that observed for the $20 \% \mathrm{w} / \mathrm{w} \mathrm{IL}+0.5 \% \mathrm{w} / \mathrm{w}$ $\mathrm{D}_{2} \mathrm{O}$ solution at $0 \mathrm{~V}$. Similarly, the high SLD of the maximum in this profile is indicative of a $\mathrm{D}_{2} \mathrm{O}$-rich layer, which forms atop the inner layer in response to the positive surface potential. At $-1.5 \mathrm{~V}$, this partitioning of the interface is reversed and a 2 layer structure recovered, in which the SLD of the inner layer is significantly decreased, indicating an increase in cations at the electrode interface.

Overall, the behaviours at the higher water concentration can be seen to be largely similar to the lower water content solution $\left(0.5 \% \mathrm{D}_{2} \mathrm{O}\right)$. The SLD profiles thus suggest that the IL ions adopt a crowding structure at the electrode interface. Likewise, the SLD profiles also reveal that the interfacial region is significantly thinner than that observed for the dry solution ( $c f$. Fig. $4 \mathrm{~b}$ inset), further indicating a local increase in effective dielectric constant. ${ }^{24}$ However, it can be seen that the effects of applied potential are more strongly determined by the potential bias, than its magnitude, suggesting a more binary responsivity.

\subsection{Tribotronic friction measurements: effect of water in $20 \% \mathrm{w} / \mathrm{w}\left[\mathrm{P}_{6,6,6,14}\right][\mathrm{BMB}]$}

Nanoscale tribotronic friction force measurements were conducted between a sharp Si tip and an electrified gold thin film in $20 \% \mathrm{w} / \mathrm{w}$ solution of $\left[\mathrm{P}_{6,6,6,14}\right][\mathrm{BMB}]$ in PC under dry $(\mathrm{Ar})$ and ambient conditions (R.H. $=22 \%$; $c f$. Section 2.4). The results for 

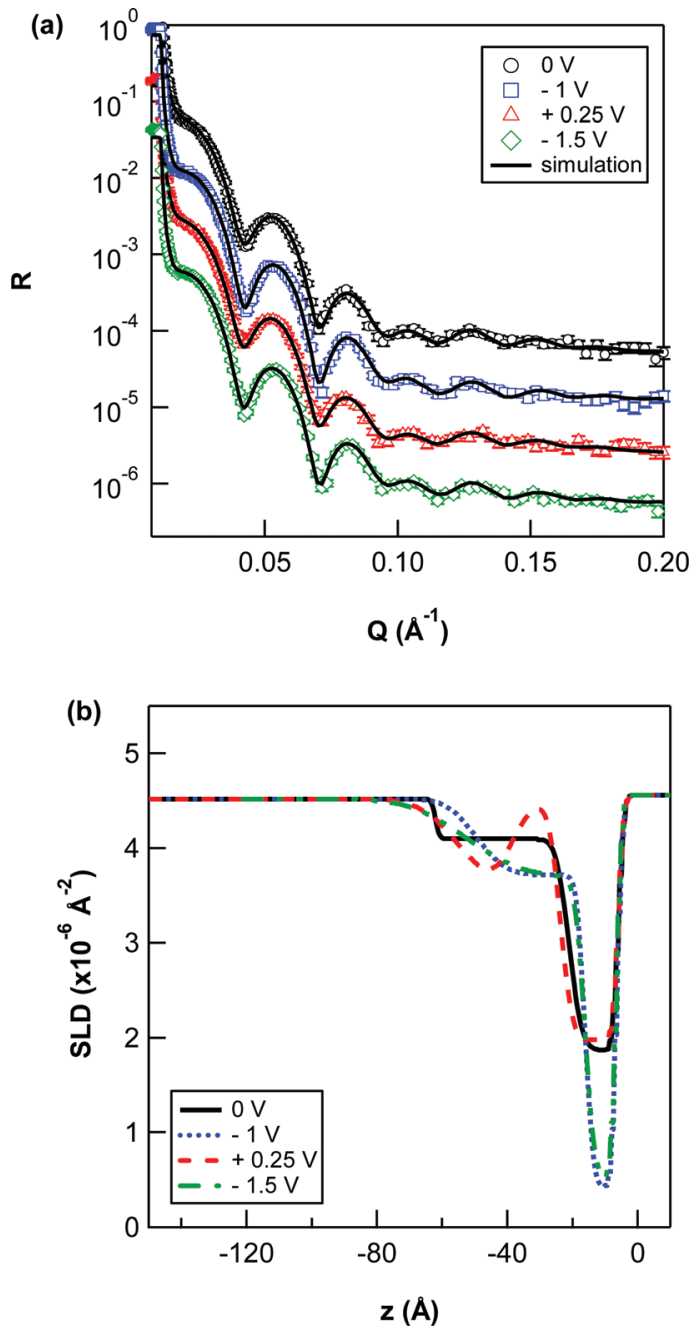

Fig. 5 (a) Reflectivity of $20 \% w / w\left[P_{6,6,6,14}\right][B M B]$ in $P C$ with $1 \% D_{2} O$ at gold electrode surface for different applied potentials (indicated in the legend). The symbols show experimental data. The solid lines represent simulated reflectivity obtained from SLD model fits. The reflectivity datasets and simulated fits have been offset in the $y$-axis for clarity. (b) Corresponding model SLD profiles to the fits to the reflectivity curves. The potentials were applied as listed in the legend.

the dry condition ${ }^{21}$ have been reported previously, but are shown here for comparison. Friction was measured during loading and unloading between 0 to $250 \mathrm{nN}$ at three different sliding velocities $\left(1,6,12 \mu \mathrm{m} \mathrm{s}^{-1}\right)$. For all conditions studied, friction increased with applied load (cf. Fig. 6a) and did not show a strong dependence on sliding velocity ( $c f$. Fig. 6a inset). In addition to the friction measurements, normal forcedistance curves were performed for each solution and potential condition to probe the interfacial structuring and overall nature of the interaction between the gold electrode surface and the AFM tip ( $c f$. ESI, $\dagger$ Fig. S7).

Consistent with previous NR measurements for a dry $20 \% \mathrm{w} / \mathrm{w}$ solution of the IL ( $c f$. Fig. 3b inset), the force-distance curves suggest a more diffuse structuring towards positive potentials, which from the NR SLD models can be attributed to a larger fraction of the anion (and possibly the solvent) at the interface.
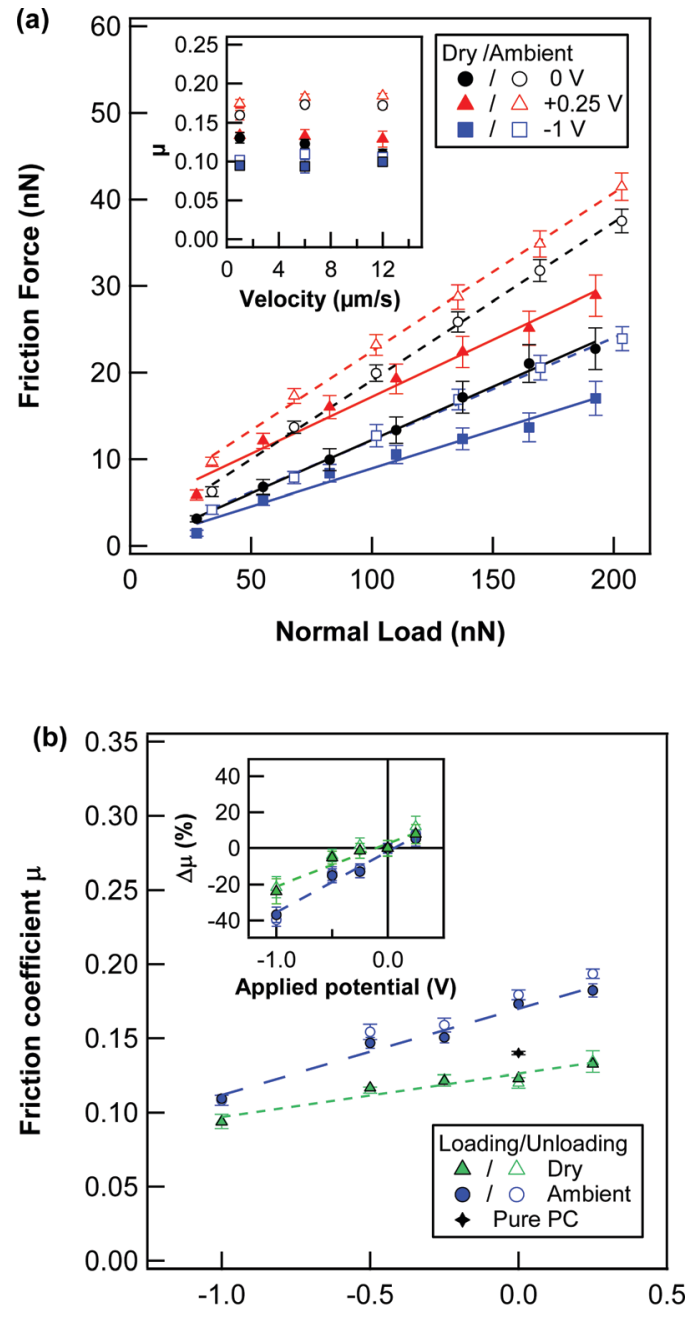

Applied potential (V)

Fig. 6 (a) Friction force as a function of normal force in a solution of $20 \% \mathrm{w} / \mathrm{w}$ $\left[P_{6,6,6,14}\right][B M B]$ in $P C$ on a gold electrode surface at three applied potentials $(0,-1$ and $+0.25 \mathrm{~V})$ in dry (filled symbols) and ambient (open symbols) conditions upon loading at a velocity of $6 \mu \mathrm{m} \mathrm{s}^{-1}$. The solid and dashed lines represent the linear fits to loading datasets used obtain corresponding friction coefficients for dry and ambient conditions, respectively. (b) Friction coefficient $\mu$ as a function of applied potential during loading and unloading for dry and ambient conditions at a velocity of $6 \mu \mathrm{m} \mathrm{s}^{-1}$. The inset shows the same data but plotted as a percentage of the friction coefficient at $\mathrm{O}(\Delta \mu \%)$ for unloading and loading, respectively. The friction coefficient $\mu$ measured for pure PC is shown for comparison.

Conversely, towards negative potentials, a more adhesive, shortranged interaction was measured. Consistent with the thinner and lower SLD interfacial layer observed in the NR SLD profiles, this interaction can be attributed to a more compact packing and higher fraction of the cation at the gold interface. ${ }^{21}$

For the same IL solution under ambient conditions (R.H. $22 \%$ ), in agreement with the NR SLD profiles for the $20 \% \mathrm{w} / \mathrm{w}$ IL solutions with added $\mathrm{D}_{2} \mathrm{O}(c f$. Fig. 4 and 5), the AFM forcedistance curves suggest an overall thinning of the interface upon exposure to ambient water, as the range of the interaction force is reduced. Moreover, the adhesive contribution to the 
interaction force, associated with an increase in the fraction of cations fraction at the interface in the dry solution, is larger, especially towards negative potentials. Such observations are consistent with the deeper SLD minimum observed in the NR SLD profiles for the IL solution with added $\mathrm{D}_{2} \mathrm{O}$ by $\mathrm{NR}$ and seem to be a result of the tendency of the IL ions to partition into more polar and non-polar regions upon exposure to small amounts of water.

For all potentials, the friction was higher for ambient conditions and more hysteretic between loading/unloading conditions, particularly towards positive potentials ( $c f$. Fig. 6b). Similar increases in friction have been reported previously for pure ILs when exposed to humidity ${ }^{7}$ and attributed to weaker interactions between the IL ions in the presence of water, reducing the loadbearing capability of the IL boundary layers under applied load. Meanwhile, in the pure solvent, the friction coefficient (measured at OCP) was found to be comparable for both dry and ambient conditions (ESI; $\dagger c f$. Fig. S8).

To the best of the authors' knowledge, the effect of water on the tribological properties of IL-solvent mixtures has not been studied previously. However, at a very dilute IL concentration $(0.01 \% \mathrm{~mol})$, it has been shown that IL boundary layers will rupture at low applied loads. ${ }^{46}$ Analogously, such behaviour was attributed to a weak interaction between the IL ions as a result of their low adsorption density.

Previous AFM studies have shown the friction of ILs and their solutions ${ }^{19,21}$ can be actively controlled through potential. Yet, the effect of water on the tribotronic lubrication of ILs and their solutions has not been investigated. For all conditions, the application of a negative potential resulted in lower friction, whilst friction increased towards positive potentials ( $c f$. Fig. 6a), whereby the friction coefficient varied approximately linearly with applied potential ( $c f$. Fig. 6b).

Such tribotronic behaviour has been reported previously for differently concentrated solutions of the same IL in PC under dry conditions. ${ }^{21}$ The observed changes in the IL lubrication are attributed to differing populations of cation and anions depending on the applied electric field, as also demonstrated by the NR measurements presented here ( $c f$. Section 3.1). As suggested by Cooper et al., ${ }^{18}$ the lubricious nature of the tetraalkylphosphonium cation is likely to originate from its long alkyl chains which facilitate the formation of robust interfacial layers, through van der Waals forces between the alkyl chains, which at positive potentials are disrupted by the small and sterically hindered IL anions.

Although the overall friction was higher in ambient conditions, the responsivity of the IL solution was clearly enhanced by the presence of water ( $c f$. Fig. $6 \mathrm{~b}$ inset). The NR results presented here for the same bulk IL concentration and a lower water concentration $(0.5 \% \mathrm{w} / \mathrm{w} ; c f$. Section 3.1.2), suggest such enhanced tribotronic behaviours are associated with crowding structures in the near interface region and intrinsic structuring properties of the individual ions. It thus appears that presence of some low levels of water may be beneficial in terms of enhanced electroresponsivity. We note, however, that additional measurements over a wider potential range ( $c f$. ESI, $\dagger$ Fig. S6) led to a dramatic reduction in friction at high positive potentials, which was also hysteretic. This suggests either that the electrochemical window of the IL solution is markedly reduced in ambient conditions for positive potentials, or that the presence of water leads to increased tribochemical reactions of the anion. This effect was completely absent under the dry conditions, where the linear trend seen in Fig. 6b was found to continue up to $+1 \mathrm{~V}(c f$. ESI, $\dagger$ Fig. S6).

\section{Summary and conclusions}

In real tribological lubricant systems complete isolation from ambient water is usually impossible and trace amounts of water are often an unavoidable impurity. Unlike other lubricants, ILs also present the possibility of active friction by changing the surface charge via external fields to modify the boundary layer structure; therefore understanding the role of water on the electroactive structuring of the IL is important.

Here we have studied the interfacial structuring of a novel halogen-free IL at a gold interface using NR. The NR measurements are compared with recent measurements for the same solutions but in a dry environment. In order to probe the effect of water on the tribological properties of the IL mixtures, complementary nanotribotronic AFM measurements for one IL concentration are also compared for dry and ambient conditions.

Overall, the NR results presented demonstrate that the electro-induced interfacial structuring of the studied IL is strongly affected by the bulk IL and water concentration. At the dilute IL concentration $(5 \% \mathrm{w} / \mathrm{w})$, the presence of water leads to a swelling of the interfacial region and anisotropy with applied potential, not observed for the dry solution. Conversely, for a concentrated IL solution $(20 \% \mathrm{w} / \mathrm{w})$, the presence of water in the interfacial region results in a partitioning of the interfacial region into distinguishable layers, which suggests the formation of a crowding structuring.

Although, not directly comparable, the behaviours observed for the concentrated $(20 \% \mathrm{w} / \mathrm{w})$ solution are largely consistent with previous theoretical predictions for low water contents in pure IL systems at charged interfaces. Furthermore, the results for the dilute solution $(5 \% \mathrm{w} / \mathrm{w})$, share similarities with predictions for higher water fractions. Such observations thus point towards the relative ratio of ion pairs to water molecules playing a key role in the behaviour of the solutions, however, the comparable dielectric properties of the solvent to that of water is also likely to play a role.

For the same bulk IL concentration $(20 \% \mathrm{w} / \mathrm{w})$, the lubricating performance of the IL in PC is clearly reduced in the presence of ambient water, compared to that in a dry Argon atmosphere. Partitioning of the IL ions within the interfacial region with exposure to water, as well as an overall thinning of the interfacial region, as determined by NR, appears to lower their load carrying capacity. Nonetheless, irrespective of water content, the measured friction coefficient is a linear function of the applied potential, whereby more negative potentials lead to 
lower friction. The presence of low levels of water is thus not necessarily a detrimental factor for tribotronic control in ILs. It remains to be seen whether the increasing stratification and interfacial water improve tribochemical or wear behaviour. This will be the focus of future studies.

\section{Conflicts of interest}

There are no conflicts to declare.

\section{Acknowledgements}

We acknowledge the Knut and Alice Wallenberg Foundation (Project No. KAW2012.0078), the Swedish Research Council (Project No. 2018-05017) and the Swedish Foundation for Strategic Research (Project No. EM16-0013) for their financial support. MR also acknowledges support from the Swedish Research council, VR (Project No. 2017-04080). We gratefully acknowledge the Science and Technology Facilities Council (STFC) for access to neutron beamtime at ISIS (Experiment RB1810114; 10.5286/ISIS.E.RB1810114), the provision of sample preparation and INTER facilities. GP would also like to thank Matthew Fielden for his help with the atomic force microscopy (AFM) measurements at the Nanofabrication facility at the Albanova University Center, Stockholm, Sweden. Dr Manishkumar Shimpi (Luleå University of Technology) is gratefully acknowledged for the synthesis of the IL.

\section{References}

1 S. Glavatskih and E. Höglund, Tribotronics-Towards active tribology, Tribol. Int., 2008, 41(9-10), 934-939.

2 J. Friedl, I. I. E. Markovits, M. Herpich, G. Feng, A. A. Kornyshev and U. Stimming, Interface between an $\mathrm{Au}(111)$ Surface and an Ionic Liquid: The Influence of Water on the Double-Layer Capacitance, ChemElectroChem, 2017, 4(1), 216-220.

3 L. A. Jurado, H. Kim, A. Rossi, A. Arcifa, J. K. Schuh, N. D. Spencer, C. Leal, R. H. Ewoldt and R. M. EspinosaMarzal, Effect of the environmental humidity on the bulk, interfacial and nanoconfined properties of an ionic liquid, Phys. Chem. Chem. Phys., 2016, 18(32), 22719-22730.

4 J. Jacquemin, P. Husson, A. A. H. Padua and V. Majer, Density and viscosity of several pure and water-saturated ionic liquids, Green Chem., 2006, 8(2), 172-180.

5 J. A. Widegren, A. Laesecke and J. W. Magee, The effect of dissolved water on the viscosities of hydrophobic roomtemperature ionic liquids, Chem. Commun., 2005, 1610-1612, DOI: 10.1039/B417348A(12).

6 A. Arcifa, A. Rossi, S. N. Ramakrishna, R. Espinosa-Marzal, A. Sheehan and N. D. Spencer, Lubrication of Si-Based Tribopairs with a Hydrophobic Ionic Liquid: The Multiscale Influence of Water, J. Phys. Chem. C, 2018, 122(13), 7331-7343.
7 R. M. Espinosa-Marzal, A. Arcifa, A. Rossi and N. D. Spencer, Microslips to "Avalanches" in Confined, Molecular Layers of Ionic Liquids, J. Phys. Chem. Lett., 2014, 5(1), 179-184.

8 R. M. Espinosa-Marzal, A. Arcifa, A. Rossi and N. D. Spencer, Ionic Liquids Confined in Hydrophilic Nanocontacts: Structure and Lubricity in the Presence of Water, J. Phys. Chem. C, 2014, 118(12), 6491-6503.

9 A. M. Smith, M. A. Parkes and S. Perkin, Molecular Friction Mechanisms Across Nanofilms of a Bilayer-Forming Ionic Liquid, J. Phys. Chem. Lett., 2014, 5(22), 4032-4037.

10 M. Taher, F. U. Shah, A. Filippov, P. de Baets, S. Glavatskih and O. N. Antzutkin, Halogen-free pyrrolidinium bis(mandelato)borate ionic liquids: some physicochemical properties and lubrication performance as additives to polyethylene glycol, RSC Adv., 2014, 4(58), 30617-30623.

11 F. U. Shah, S. Glavatskih and O. N. Antzutkin, Boron in Tribology: From Borates to Ionic Liquids, Tribol. Lett., 2013, 51(3), 281-301.

12 R. F. de Souza, J. C. Padilha, R. S. Gonçalves and J. RaultBerthelot, Dialkylimidazolium ionic liquids as electrolytes for hydrogen production from water electrolysis, Electrochem. Commun., 2006, 8(2), 211-216.

13 M. Radiom, Ionic liquid-solid interface and applications in lubrication and energy storage, Curr. Opin. Colloid Interface Sci., 2019, 39, 148-161.

14 Z. Wang, H. Li, R. Atkin and C. Priest, Influence of Water on the Interfacial Nanostructure and Wetting of [Rmim][NTf2] Ionic Liquids at Mica Surfaces, Langmuir, 2016, 32(35), 8818-8825.

15 R. G. Horn, D. F. Evans and B. W. Ninham, Double-layer and solvation forces measured in a molten salt and its mixtures with water, J. Phys. Chem., 1988, 92(12), 3531-3537.

16 J. A. Smith, O. Werzer, G. B. Webber, G. G. Warr and R. Atkin, Surprising Particle Stability and Rapid Sedimentation Rates in an Ionic Liquid, J. Phys. Chem. Lett., 2010, 1(1), 64-68.

17 S. Kawada, E. Kodama, K. Sato, S. Ogawa, M. Watanabe, H. Okubo and S. Sasaki, Effect of water on the interfacial structures of room-temperature ionic liquids, Surf. Interface Anal., 2019, 51(1), 17-20.

18 P. Cooper, H. Li, M. W. Rutland, G. Webber and R. Atkin, Tribotronic control of friction in oil-based lubricants with ionic liquid additives, Phys. Chem. Chem. Phys., 2016, 18(34), 23657-23662.

19 J. Sweeney, F. Hausen, R. Hayes, G. B. Webber, F. Endres, M. W. Rutland, R. Bennewitz and R. Atkin, Control of Nanoscale Friction on Gold in an Ionic Liquid by a Potential-Dependent Ionic Lubricant Layer, Phys. Rev. Lett., 2012, 109(15), 155502.

20 H. Li, R. J. Wood, M. W. Rutland and R. Atkin, An ionic liquid lubricant enables superlubricity to be "switched on" in situ using an electrical potential, Chem. Commun., 2014, 50(33), 4368-4370.

21 G. A. Pilkington, A. Oleshkevych, P. P. Carrasco, S. Watanabe, M. Radiom, A. B. Reddy, A. Vorobiev, S. Glavatskih and M. W. Rutland, Electroresponsive structuring and friction of 
a non-halogenated ionic liquid in a polar solvent: Effect of concentration, Phys. Chem. Chem. Phys., 2020, 22, 19162-19171.

22 G. A. Pilkington, K. Harris, E. Bergendal, A. B. Reddy, G. K. Palsson, A. Vorobiev, O. N. Antzutkin, S. Glavatskih and M. W. Rutland, Electro-responsivity of ionic liquid boundary layers in a polar solvent revealed by neutron reflectance, J. Chem. Phys., 2018, 148(19), 193806.

23 G. Feng, X. Jiang, R. Qiao and A. A. Kornyshev, Water in Ionic Liquids at Electrified Interfaces: The Anatomy of Electrosorption, ACS Nano, 2014, 8(11), 11685-11694.

24 O. Y. Fajardo, F. Bresme, A. A. Kornyshev and M. Urbakh, Water in Ionic Liquid Lubricants: Friend and Foe, ACS Nano, 2017, 11(7), 6825-6831.

$25 \mathrm{~K}$. Motobayashi and M. Osawa, Potential-dependent condensation of Water at the Interface between ionic liquid [BMIM][TFSA] and an Au electrode, Electrochem. Commun., 2016, 65, 14-17.

26 Y. Zhong, J. Yan, M. Li, L. Chen and B. Mao, The Electric Double Layer in an Ionic Liquid Incorporated with Water Molecules: Atomic Force Microscopy Force Curve Study, ChemElectroChem, 2016, 3(12), 2221-2226.

27 P. Rohlmann, B. Munavirov, I. Furó, O. Antzutkin, M. W. Rutland and S. Glavatskih, Non-halogenated Ionic Liquid Dramatically Enhances Tribological Performance of Biodegradable Oils, Front. Chem., 2019, 7, 98.

28 N. Hjalmarsson, D. Wallinder, S. Glavatskih, R. Atkin, T. Aastrup and M. W. Rutland, Weighing the surface charge of an ionic liquid, Nanoscale, 2015, 7(38), 16039-16045.

29 N. Hjalmarsson, E. Bergendal, Y.-L. Wang, B. Munavirov, D. Wallinder, S. Glavatskih, T. Aastrup, R. Atkin, I. Furó and M. W. Rutland, Electro-Responsive Surface Composition and Kinetics of an Ionic Liquid in a Polar Oil, Langmuir, 2019, 35(48), 15692-15700.

30 F. U. Shah, S. Glavatskih, D. R. MacFarlane, A. Somers, M. Forsyth and O. N. Antzutkin, Novel halogen-free chelated orthoborate-phosphonium ionic liquids: synthesis and tribophysical properties, Phys. Chem. Chem. Phys., 2011, 13(28), 12865-12873.

31 Y.-L. Wang, M. Golets, B. Li, S. Sarman and A. Laaksonen, Interfacial Structures of Trihexyltetradecylphosphoniumbis(mandelato)borate Ionic Liquid Confined between Gold Electrodes, ACS Appl. Mater. Interfaces, 2017, 9(5), 4976-4987.

32 A. M. Smith, A. A. Lee and S. Perkin, The Electrostatic Screening Length in Concentrated Electrolytes Increases with Concentration, J. Phys. Chem. Lett., 2016, 7(12), 2157-2163.

33 O. Arnold, J. C. Bilheux, J. M. Borreguero, A. Buts, S. I. Campbell, L. Chapon, M. Doucet, N. Draper, R. Ferraz Leal, M. A. Gigg, V. E. Lynch, A. Markvardsen, D. J. Mikkelson, R. L. Mikkelson, R. Miller, K. Palmen, P. Parker, G. Passos, T. G. Perring, P. F. Peterson, S. Ren, M. A. Reuter, A. T. Savici, J. W. Taylor, R. J. Taylor, R. Tolchenov, W. Zhou and
J. Zikovsky, Mantid-Data analysis and visualization package for neutron scattering and $\mu \mathrm{SR}$ experiments, Nucl. Instrum. Methods Phys. Res., Sect. B, 2014, 764, 156-166.

34 M. Björck and G. Andersson, GenX: an extensible X-ray reflectivity refinement program utilizing differential evolution, J. Appl. Crystallogr., 2007, 40(6), 1174-1178.

35 P. Croce and L. Névot, Étude des couches minces et des surfaces par réflexion rasante, spéculaire ou diffuse, de rayons X, Revue de physique appliquée, 1976, 11(1), 113-125.

36 A. Feiler, P. Attard and I. Larson, Calibration of the torsional spring constant and the lateral photodiode response of frictional force microscopes, Rev. Sci. Instrum., 2000, 71(7), 2746-2750.

37 G. Bogdanovic, A. Meurk and M. W. Rutland, Tip friction-torsional spring constant determination, Colloids Surf., B, 2000, 19(4), 397-405.

38 R. Álvarez-Asencio, E. Thormann and M. W. Rutland, Note: Determination of torsional spring constant of atomic force microscopy cantilevers: Combining normal spring constant and classical beam theory, Rev. Sci. Instrum., 2013, 84(9), 096102.

39 T. Pettersson, N. Nordgren, M. W. Rutland and A. Feiler, Comparison of different methods to calibrate torsional spring constant and photodetector for atomic force microscopy friction measurements in air and liquid, Rev. Sci. Instrum., 2007, 78(9), 093702.

40 Y.-L. Wang, S. Sarman, L. Kloo, O. N. Antzutkin, S. Glavatskih and A. Laaksonen, Solvation structures of water in trihexyltetradecylphosphonium-orthoborate ionic liquids, J. Chem. Phys., 2016, $145(6), 064507$.

41 M. V. Fedorov, N. Georgi and A. A. Kornyshev, Double layer in ionic liquids: The nature of the camel shape of capacitance, Electrochem. Commun., 2010, 12(2), 296-299.

42 S. Watanabe, G. Pilkington, A. Oleshkevych, P. P. Carrasco, M. Radiom, R. Welbourn, S. Glavatskih and M. W. Rutland, Interfacial Structure of Non-halogenated Imidazolium Ionic Liquids at Charged Surfaces: Effect of Alkyl Chain Length, Phys. Chem. Chem. Phys., 2020, 22, 8450-8460.

43 C. Y. Peñalber, G. A. Baker and S. Baldelli, Sum frequency generation spectroscopy of imidazolium-based ionic liquids with cyano-functionalized anions at the solid salt-liquid interface, J. Phys. Chem. B, 2013, 117(19), 5939-5949.

44 M. Z. Bazant, B. D. Storey and A. A. Kornyshev, Double layer in ionic liquids: Overscreening versus crowding, Phys. Rev. Lett., 2011, 106(4), 046102.

45 L. A. Jurado and R. M. Espinosa-Marzal, Insight into the Electrical Double Layer of an Ionic Liquid on Graphene, Sci. Rep., 2017, 7(1), 4225.

$46 \mathrm{H}$. Li, A. E. Somers, M. W. Rutland, P. C. Howlett and R. Atkin, Combined Nano-and Macrotribology Studies of Titania Lubrication Using the Oil-Ionic Liquid Mixtures, ACS Sustainable Chem. Eng., 2016, 4(9), 5005-5012. 